\title{
Simultaneous bilateral thoracoscopic blebs excision reduces contralateral recurrence in patients undergoing operation for ipsilateral primary spontaneous pneumothorax
}

\author{
Yu-Wei Liu, MD, ${ }^{\mathrm{a}, \mathrm{b}}$ Po-Chih Chang, MD, ${ }^{\mathrm{a}}$ Shun-Jen Chang, MD, ${ }^{\mathrm{c}}$ Hung-Hsing Chiang, MD, \\ Hsien-Pin Li, MD, ${ }^{a, b}$ and Shah-Hwa Chou, MD ${ }^{a, d, e}$
}

\section{ABSTRACT}

Objective: Contralateral recurrence in patients with primary spontaneous pneumothorax is approximately $15 \%$. If positive for blebs, the recurrence rate increases to $26 \%$. This study seeks to determine whether simultaneous contralateral video-assisted thoracic surgery blebs excision would effectively lower the contralateral incidence of pneumothorax in patients undergoing surgery for ipsilateral primary spontaneous pneumothorax.

Methods: Between January 2009 and December 2015, 335 patients with primary spontaneous pneumothorax, surgically treated in a single institution, were retrospectively studied. The median follow-up was 75 (50-99) months. All patients received video-assisted thoracic surgery blebectomy/bullectomy with pleural abrasions. They were classified into 3 groups: (1) ipsilateral video-assisted thoracic surgery without contralateral blebs/bullae included 142 patients with ipsilateral primary spontaneous pneumothorax without contralateral blebs/bullae only receiving ipsilateral video-assisted thoracic surgery; (2) ipsilateral video-assisted thoracic surgery with contralateral blebs/ bullae included 123 patients with ipsilateral primary spontaneous pneumothorax with contralateral blebs/bullae receiving only ipsilateral video-assisted thoracic surgery; and (3) bilateral video-assisted thoracic surgery with contralateral blebs/bullae included 70 patients with ipsilateral primary spontaneous pneumothorax with contralateral blebs/bullae receiving 1-stage bilateral video-assisted thoracic surgery. Demographic data, perioperative details, recurrence patterns, recurrence-free survivals, and risk factors were compared.

Results: The percentage of contralateral recurrence for the ipsilateral video-assisted thoracic surgery without contralateral blebs/bullae, ipsilateral video-assisted thoracic surgery with contralateral blebs/bullae, and bilateral video-assisted thoracic surgery with contralateral blebs/bullae groups differed significantly $(0.7 \%, 14.6 \%$, and $2.9 \%$, respectively; $P=.002)$. Multivariate analysis using the Cox proportional hazard model revealed that age less than 18 years (hazard ratio, $2.71 ; 95 \%$ confidence interval, $1.14-6.44 ; P=.024$ ) and ipsilateral video-assisted thoracic surgery with contralateral blebs/bullae (hazard ratio, $22.13,95 \%$ confidence interval, $2.96-165, P=.003$ ) were predictors of contralateral recurrence, of which recurrence-free survival was notably different among groups as determined by Kaplan-Meier analysis $(P<.0001)$.

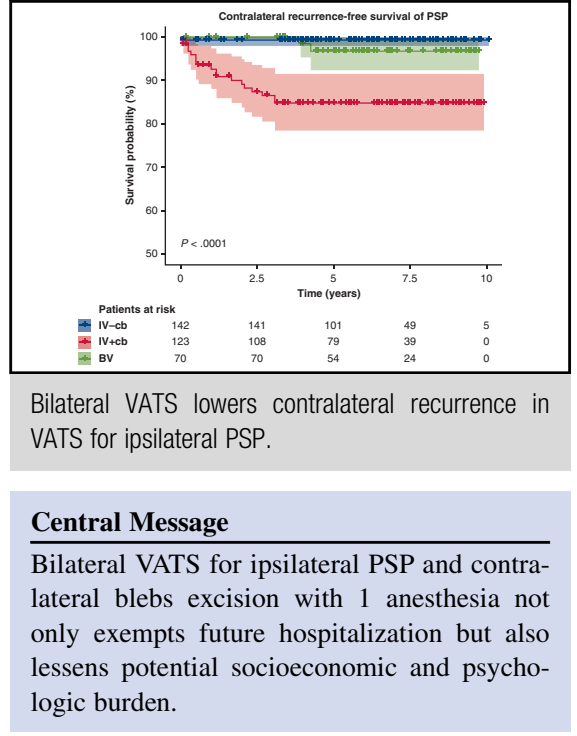

\section{Perspective}

This is the largest study related to bilateral VATS for ipsilateral PSP and contralateral blebectomy with a median follow-up of 75 months For patients exhibiting contralateral blebs, the $\mathrm{BV}$ group had significantly lower contralateral recurrence than the IV $+\mathrm{cb}$ group $(2.9 \%$ vs $14.6 \%$ ). In VATS for ipsilateral PSP, contralateral blebectomy with the same anesthesia notably lowers future contralateral recurrence.

See Commentary on page 1128 .

\footnotetext{
From the ${ }^{\mathrm{a} D i v i s i o n}$ of Thoracic Surgery, Department of Surgery, Kaohsiung Medical University Hospital; ${ }^{b}$ Graduate Institute of Clinical Medicine, College of Medicine, and ${ }^{\mathrm{d}}$ Department of Respiratory Therapy, College of Medicine, Kaohsiung Medical University, Kaohsiung, Taiwan; ${ }^{\mathrm{C}}$ Department of Kinesiology, Health and Leisure Studies, National University of Kaohsiung, Kaohsiung, Taiwan; ${ }^{\mathrm{e} D e}$ partment of Surgery, Pingtung Hospital, Ministry of Health and Welfare, Pingtung, Taiwan.
}

Received for publication May 28, 2019; revisions received July 23, 2019; accepted for publication Aug 1, 2019; available ahead of print Oct 9, 2019.

Address for reprints: Shah-Hwa Chou, MD, No. 100, Tzyou 1st Rd, Kaohsiung 80756, Taiwan (E-mail: shhwch@cc.kmu.edu.tw).

$0022-5223 / \$ 36.00$

Copyright (C) 2019 by The American Association for Thoracic Surgery

https://doi.org/10.1016/j.jtcvs.2019.08.009 


\section{Abbreviations and Acronyms \\ BMI = body mass index \\ $\mathrm{BV}=$ bilateral video-assisted thoracic surgery with contralateral blebs/bullae \\ $\mathrm{CI}=$ confidence interval \\ HR = hazard ratio \\ HRCT $=$ high-resolution computed tomography \\ $\mathrm{IQR}=$ interquartile range \\ $\mathrm{IV}$-cb = ipsilateral video-assisted thoracic surgery without contralateral blebs/bullae \\ $\mathrm{IV}+\mathrm{cb}=$ ipsilateral video-assisted thoracic surgery with contralateral blebs/bullae \\ PSP = primary spontaneous pneumothorax \\ VATS $=$ video-assisted thoracic surgery}

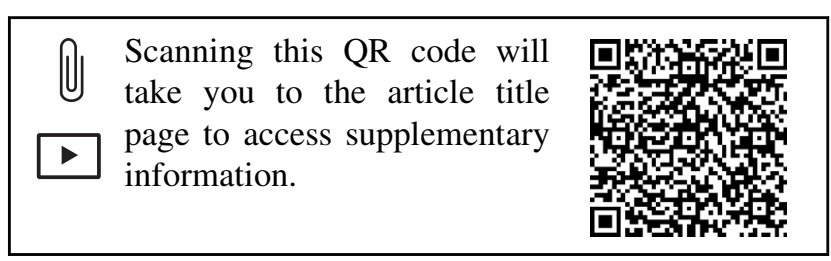

Conclusions: Simultaneous contralateral blebectomy in patients with primary spontaneous pneumothorax receiving ipsilateral video-assisted thoracic surgery significantly lowered future contralateral recurrence. (J Thorac Cardiovasc Surg 2020;159:1120-7)

Primary spontaneous pneumothorax (PSP) is a common benign disease affecting adolescents and young adults. According to statistics from Taiwan's national populationbased cohort study in 2013, the annual incidence is approximately 7.18 per 100,000 (male/female odds ratio: 9.7), ${ }^{1}$ which is comparable to the data reported in Western countries. $^{2}$

Recurrence has remained an issue in patients with PSP, resulting in high stress for patients and socioeconomic burden for healthcare systems. ${ }^{3,4}$ The primary goal of pneumothorax treatment is to prevent future recurrence. One of the widely accepted theories for the recurrence of PSP is the rupture of pulmonary blebs or bullae; thus, the presence of these air-containing lesions on high-resolution computed tomography (HRCT) has been regarded as a significant predictor worthy of investigation. The reported incidence of blebs/bullae on HRCT ranged from $47 \%$ to $94 \%$ in the ipsilateral lung ${ }^{4-10}$ and $55 \%$ to $70 \%$ in the contralateral lung..$^{8-10}$

Conservative treatment is associated with an ipsilateral recurrence range of $16 \%$ to $52 \%$ and a contralateral recurrence rate of $5 \%$ to $15 \%,{ }^{3,11-13}$ with contralateral recurrence increasing to approximately $26 \%$ if contralateral blebs and bullae are present. ${ }^{4,7,8}$ Surgery is indisputably the most effective method to prevent recurrence. International consensus recommends surgical intervention after the first ipsilateral or contralateral recurrence, as mentioned in the leading guidelines. ${ }^{2,14,15}$ Although some literature advocates early surgery for patients presenting with blebs or bullae after a first episode of PSP, ${ }^{4,16-18}$ as well as some physicians proving the feasibility and safety of performing single-stage unilateral or bilateral video-assisted thoracic surgery (VATS) in selected patients with PSP with contralateral blebs/bullae using different techniques, ${ }^{19-24}$ the effect of contralateral bleb excision in the prevention of contralateral recurrence with the same anesthesia for ipsilateral PSP remains controversial. No study or follow-up data show the outcome or effect of the contralateral surgery.

In our earlier preliminary study, preemptive VATS treatment for the contralateral blebs/bullae reduced contralateral recurrence. ${ }^{9}$ The current study consists of a larger, new population with more skillful clinical process and longer follow-up period, in the hope of obtaining a more convincing and reliable result.

The primary end points of this study were recurrence rates (contralateral, ipsilateral, and overall) of patients who were treated as part of the ipsilateral VATS without contralateral blebs/bullae (IV-cb) group, ipsilateral VATS with contralateral blebs/bullae $(\mathrm{IV}+\mathrm{cb})$ group, or bilateral VATS with contralateral blebs/bullae (BV) group. The secondary end points were recurrence-free survivals and potential risk factors for the recurrence of pneumothorax.

\section{MATERIAL AND METHODS \\ Study Design and Patients}

After approval by the Institutional Review Board of Kaohsiung Medical University Hospital (approval KMUHIRB-E(I)-20190158), our retrospective study based on a prospective database was reviewed for 404 patients who received VATS for pneumothorax between January 2009 and December 2015 at this single medical center. As shown in Figure 1, exclusion criteria involved patients with secondary spontaneous pneumothorax (ie, chronic obstructive pulmonary disease, catamenial pneumothorax, or interstitial lung disease), age of 40 years and older, lack of chest computed tomography investigation, or lost telephone follow-up. Patients with contralateral recurrence after previous PSP and simultaneous bilateral PSP were eliminated to obtain a more uniform analysis. A total of 335 patients with PSP with complete follow-up remained after exclusion criteria. They all received preoperative HRCT scan without contrast media. Based on guidelines and consensus, ${ }^{2,14,15}$ indications for surgery at our institution predominantly included recurrent ipsilateral PSP and persistent air leaks (>3 days) after chest tube drainage. Because of existing scientific substantiation, ${ }^{4,16-18}$ VATS was also offered to patients with PSP at the first attack involving ipsilateral or contralateral blebs/bullae on HRCT. Patient demographic data, including age, gender, body mass index (BMI), smoking habit, operation time, blood loss, postoperative pain score, postoperative hospital length of stay, recurrence after surgery, and 


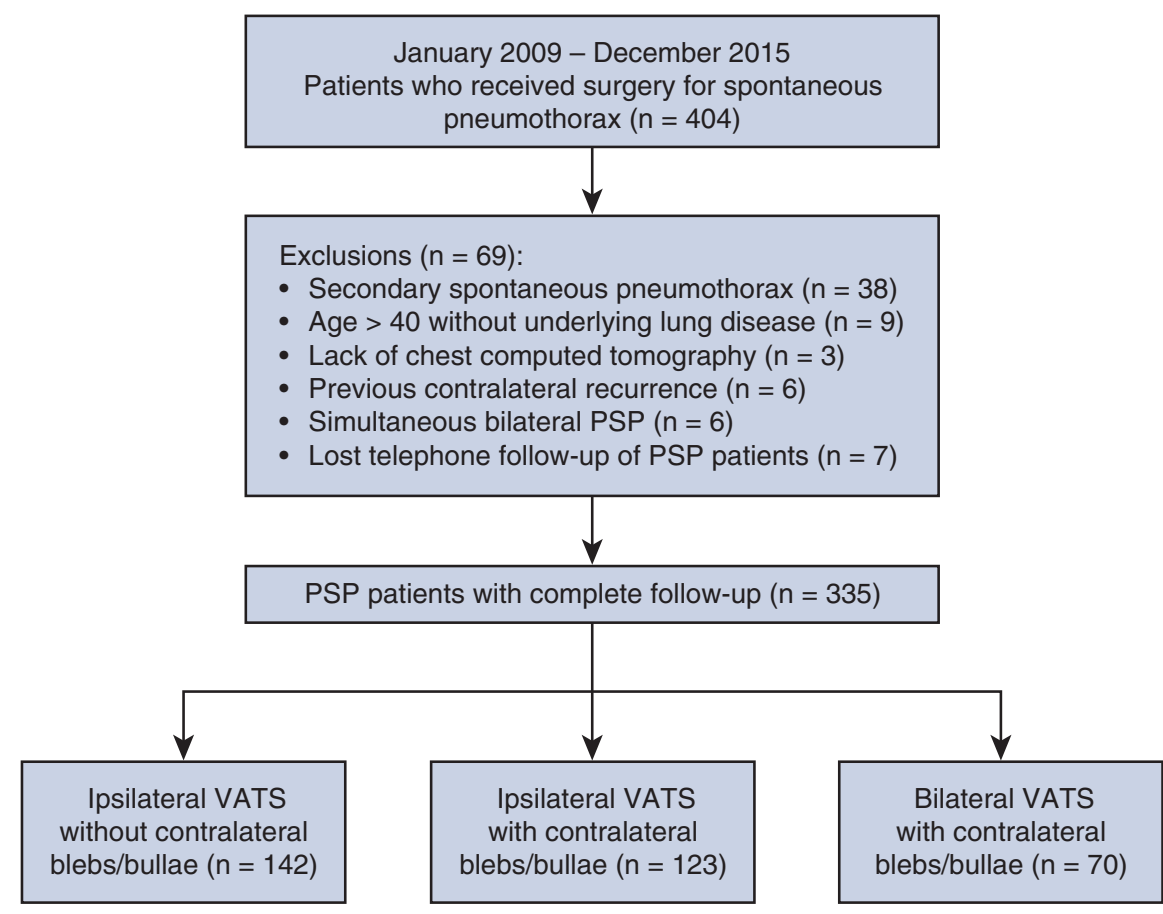

FIGURE 1. Flow diagram for patient enrollment. PSP, Primary spontaneous pneumothorax; VATS, video-assisted thoracic surgery.

follow-up times, were collected in a review of medical records and analyzed.

\section{High-Resolution Computed Tomography Protocol and Interpretation}

The HRCT was conducted during the in-hospital period after the affected lung was expanded as completely as possible. Computed tomography scan examinations were performed at our hospital using the Optima CT660 system (GE, Tokyo, Japan). Reconstruction thickness was contiguous $5 \mathrm{~mm}$ in axial, coronal, and sagittal sections. Additionally, the reconstruction thickness and interval for thin slices of axial section were 1.0 and 2.0 to $5.0 \mathrm{~mm}$, respectively. The HRCT images were carefully interpreted by at least 2 thoracic surgeons and 1 radiologist to ascertain the presence of blebs/bullae.

As reported in our previous study, ${ }^{9}$ patients presenting with contralateral blebs/bullae on HRCT had their ailment fully explained to them and their family: (1) The ipsilateral VATS had to be performed for recurrence, persistent air leak, or radiographically exhibited blebs/bullae; (2) the incidental discovery of the contralateral blebs/bullae; or (3) the likelihood and reported rate of contralateral recurrence. ${ }^{11,12}$ Contralateral VATS was proposed on the same operation but without any persuasion. Rejection or acceptance of the contralateral surgery was decided solely by the patient and their family. All the patients gave informed consent involving contralateral precautionary VATS.

\section{Operative Technique}

As reported previously, ${ }^{9}$ the operation (needlescopic VATS) was performed in the lateral decubitus position under general anesthesia using double-lumen endotracheal tube for lung isolation. The blebs/bullae were identified carefully over the entire lung surface and grasped with a 3-mm endograsper, followed by resection via the endoscopic stapler. Mechanical pleural abrasion was performed under direct view of the needlescope using curved ring forceps wrapped with a piece of Marlex mesh. The contralateral lung was operated on in a similar fashion when 1-stage bilateral VATS was scheduled. We routinely use chest tubes (24F-28F), and no regular suction is applied after surgery. Chest tube removal is based on the criteria of ensured clear pleural drainage and absence of any air leak.

\section{Follow-up}

All patients were advised to receive outpatient clinic follow-up in the event of any discomfort or suspicious symptoms of recurrence. Chest radiographs were performed for diagnosis of recurrent pneumothorax if the mentioned condition developed at any time during follow-up. HRCT scan for normal chest radiography was performed in highly suspicious cases. They were followed up by telephone interviews and medical records in February 2019 to determine the incidence of recurrence. Seven patients who could not be reached by phone were excluded from analysis of recurrence. The remaining 335 patients were contacted and reported their condition regarding PSP recurrence successfully.

\section{Statistical Analysis}

The patients were divided into 3 groups: (1) ipsilateral VATS without contralateral blebs/bullae (IV-cb); (2) ipsilateral VATS with contralateral blebs/bullae (IV+cb); and (3) bilateral VATS with contralateral blebs/ bullae (BV). Continuous variables were described by means \pm standard deviation or medians with interquartile range (IQR), and categoric variables were summarized by frequency and percentage. Continuous variables were compared with the analysis of variance or Kruskal-Wallis test, and categoric variables were compared using the chi-square test. Bonferroni correction was made for adjusting the effects of multiple comparison when the tests showed that there was a statistical difference between groups. Recurrence-free survivals and follow-up times were calculated from the date of surgery to the first event of recurrence and analyzed by the Kaplan-Meier method. Log-rank test was used to examine the differences between treatment groups. Univariate and multivariate analyses were performed with the Cox proportional hazard model to investigate risk factors 
for different recurrence pattern. All statistical analyses were performed using SAS 9.4 software (SAS Institute Inc, Cary, NC) for Windows.

\section{RESULTS}

\section{Patient Characteristics}

A total of 335 patients with PSP who had undergone VATS procedures were included over the 7-year study period. There were 142 patients in group IV-cb, 123 patients in group IV $+\mathrm{cb}$, and 70 patients in group BV. Computed tomography scans of these groups are shown in Figures E1E3. We constructed a figure demonstrating the patients with PSP receiving different surgical treatment from 2009 to 2015 (Figure 2). The average acceptance rate of the simultaneous contralateral bleb excision in our cohort study is $36 \%(70 / 193)$. The bar graph in Figure 3 demonstrates the age-stratified number of patients per treatment group. The clinical demographic data of patients regarding age, BMI, gender, and smoking show comparable findings in Table 1 .

\section{Perioperative Outcomes}

The operation data of the 3 groups are shown and compared in Table 2, which reveals statistical significance and adjusted with Bonferroni correction. The operative time and length of hospital stay were significantly longer in group $\mathrm{BV}(P<.001)$; the postoperative pain score was also higher in group BV $(P<.001)$. Complications were statistically insignificant in either group. Persistent air leak (defined as persisting for $>7$ days postoperatively) accounts for the majority, followed by pneumonia, wound infection, and hemothorax. Re-do VATS was required to treat severe persistent air leaks for 1 patient, 2 patients, and 1 patient in groups IV-cb, IV $+\mathrm{cb}$, and $\mathrm{BV}$, respectively. Follow-up periods of the 3 different groups did not differ significantly (median, 75; IQR, 50-99 months; $P=.189$ ).

\section{Primary Spontaneous Pneumothorax Recurrence Pattern}

The percentages of contralateral recurrence for groups IV-cb, IV $+\mathrm{cb}$, and BV were $0.7 \%, 14.6 \%$, and $2.9 \%$, respectively. Notably, the percentage for group $\mathrm{IV}+\mathrm{cb}$ was significantly higher than for the other 2 groups $(P=.002)$. Contralateral recurrences occurred in 21 patients. Except for 1 patient with mild pneumothorax who underwent conservative care (observation), the other 20 patients received VATS treatment. Ipsilateral recurrences occurred in 27 patients, and treatment alternatives included re-do VATS (19 patients), chest tube drainage with chemical pleurodesis (4 patients), and observation (4 patients).

\section{Analysis of Factors on Risk of Recurrence}

Table 3 shows the univariate analysis between potential risk factors and recurrences, with only age $(P=.003)$ and treatment group $(P<.001)$ reaching statistical significance. Further multivariate analysis using the Cox proportional hazard model was performed. As shown in Table E1, age less than 18 years was the independent predictor of contralateral recurrence (hazard ratio [HR], 2.71; 95\% confidence interval $[\mathrm{CI}], 1.14-6.44, P=.024)$ and ipsilateral recurrence (HR, 4.29, 95\% CI, 2.01-9.17, $P=.0002$ ). Moreover,

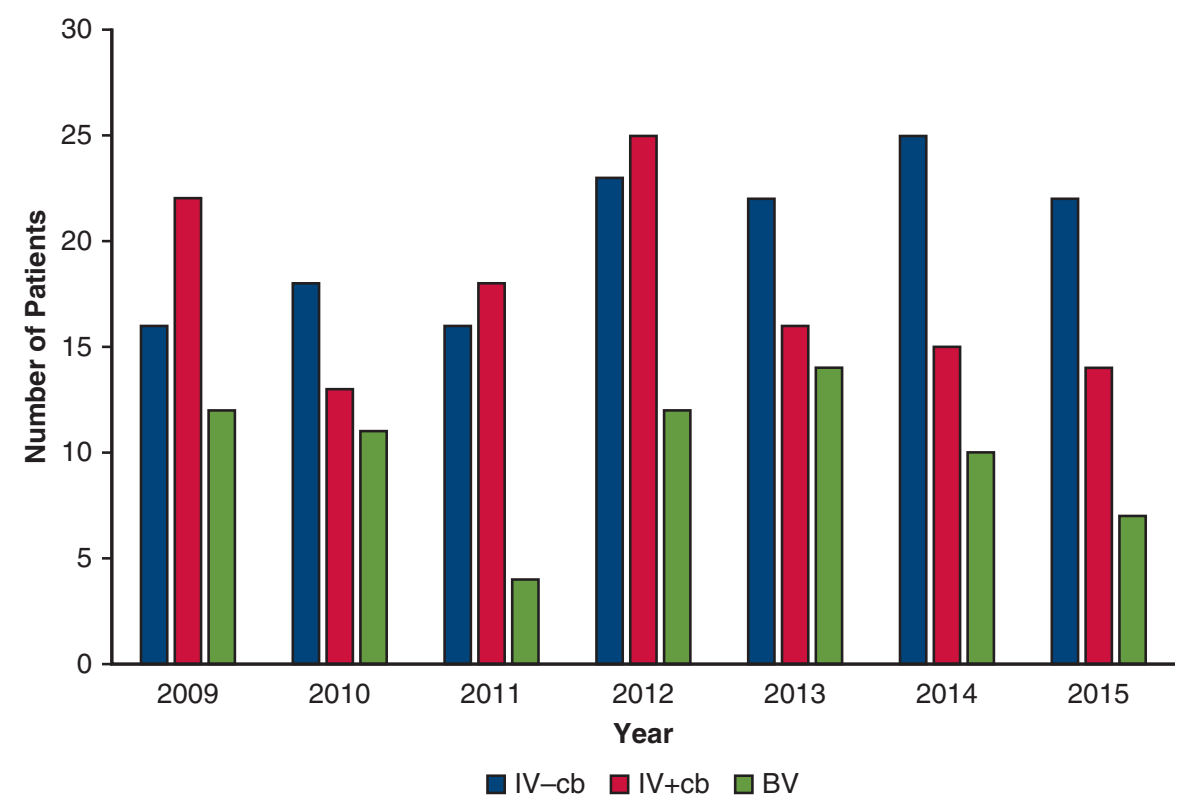

FIGURE 2. Patients with PSP treated with VATS from 2009 to 2015. $I V$ - $c b$, Ipsilateral video-assisted thoracic surgery without contralateral blebs/bullae; $I V+c b$, ipsilateral video-assisted thoracic surgery with contralateral blebs/bullae; $B V$, bilateral video-assisted thoracic surgery with contralateral blebs/ bullae. 


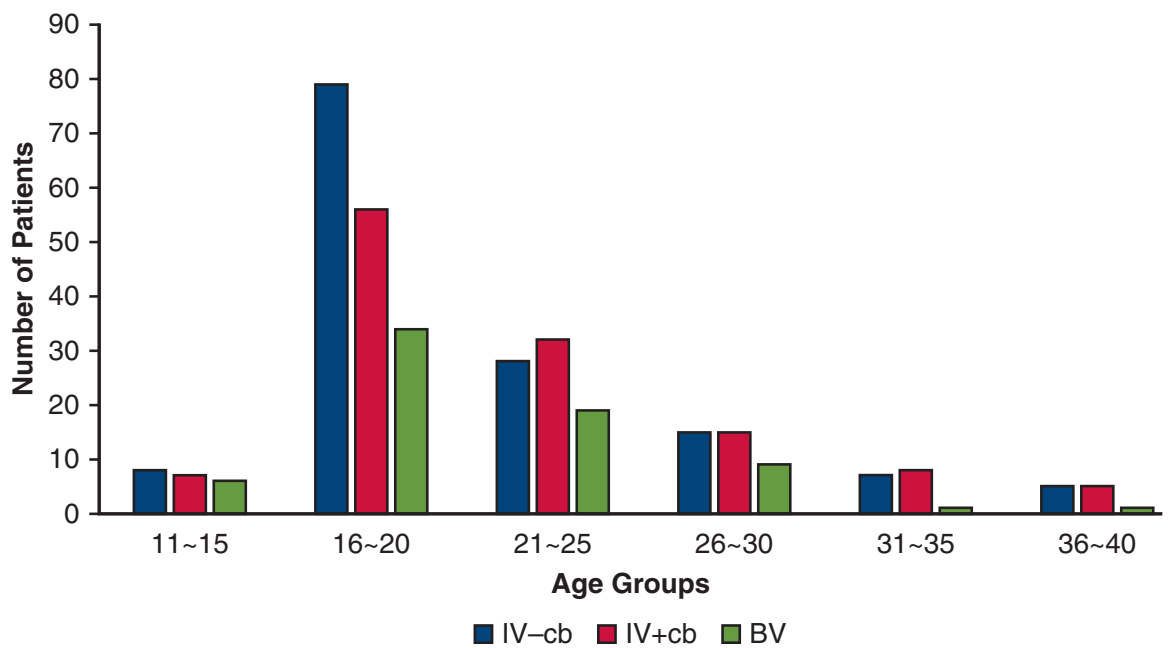

FIGURE 3. Age-stratified number of patients per groups. $I V-c b$, Ipsilateral video-assisted thoracic surgery without contralateral blebs/bullae; $I V+c b$, ipsilateral video-assisted thoracic surgery with contralateral blebs/bullae; $B V$, bilateral video-assisted thoracic surgery with contralateral blebs/bullae.

treatment group IV $+\mathrm{cb}(\mathrm{HR}, 22.13,95 \% \mathrm{CI}, 2.96-165$, $P=.003$ ) was another independent predictor of contralateral recurrence. Figure 4 demonstrates the contralateral recurrence-free survival calculated using the KaplanMeier method and compared through the log-rank test.

For patients exhibiting contralateral blebs/bullae, contralateral recurrence was significantly different between groups $\mathrm{IV}+\mathrm{cb}$ and $\mathrm{BV}$, with group $\mathrm{BV}$ showing the lower recurrence $(P<.0001)$. Moreover, overall and ipsilateral recurrence-free survivals were indicated in Figures E4 and E5.

\section{DISCUSSION}

To our knowledge, this study is currently one of the few related to contralateral blebs intervention with ipsilateral VATS for PSP. It consists of the largest population, with long-term follow-up periods. In our study over a 7-year period, 193 of 335 patients with unilateral PSP were discovered, through HRCT, to have contralateral blebs/bullae. The reported rate was $57.6 \%$, which is similar to the rate reported in the literature..$^{8-10}$ According to our results, the percentage of ipsilateral recurrence was statistically insignificant among the 3 groups. However, specifically for patients exhibiting contralateral blebs/bullae, group
BV has significantly lower contralateral recurrence than group IV + cb.

In the literature, predictability of a contralateral event after a first episode of PSP is debatable. Some researchers found that the detection of blebs/bullae through HRCT in the contralateral lung after a first episode of PSP was associated with a higher rate of contralateral recurrence. The calculated absolute risks for a contralateral recurrence in the presence of contralateral blebs/bullae among different studies were $26 \%, 26.7 \%$, and $25.8 \%$, respectively. $4,7,8$ Further, these studies reported that no patients without blebs or bullae experienced a contralateral recurrence. However, on the basis of our finding, 1 episode of contralateral recurrence occurred in group IV-cb in the third year after surgery. The patient eventually underwent VATS treatment, and small blebs were discovered intraoperatively under thoracoscopic viewing. Occult small blebs in the apical regions may be difficult to identify; thus, correct protocol and interpretation of HRCT are crucial. Kim and colleagues $^{17}$ noticed that certain patients with PSP without detectable air-containing lesions on HRCT had recurrence, and several blebs/bullae were revealed upon operation in such group. They highlighted the imperfect sensitivity of preoperative detection by HRCT and therefore

TABLE 1. Patient characteristics

\begin{tabular}{|c|c|c|c|c|}
\hline & IV-cb $(n=142)$ & $\mathrm{IV}+\mathrm{cb}(\mathrm{n}=123)$ & $\mathbf{B V}(\mathbf{n}=70)$ & $P$ value \\
\hline Age $($ mean \pm SD) $(y)$ & $21.3 \pm 5.5$ & $22.0 \pm 6.1$ & $20.6 \pm 4.7$ & .204 \\
\hline $\mathrm{BMI}($ mean $\pm \mathrm{SD})\left(\mathrm{kg} / \mathrm{m}^{2}\right)$ & $19.5 \pm 2.1$ & $19.5 \pm 2.4$ & $19.3 \pm 2.1$ & .833 \\
\hline Gender (male) & $118(83)$ & $111(90)$ & $65(93)$ & .072 \\
\hline Smoking (yes) & $25(18)$ & $27(22)$ & $20(29)$ & .229 \\
\hline
\end{tabular}

Data are presented as No. $(\%) . I V$ - $c b$, Ipsilateral video-assisted thoracic surgery without contralateral blebs/bullae; $I V+c b$, ipsilateral video-assisted thoracic surgery with contralateral blebs/bullae; $B V$, bilateral video-assisted thoracic surgery with contralateral blebs/bullae; $S D$, standard deviation; $B M I$, body mass index. 
TABLE 2. Clinical features of patients with perioperative data

\begin{tabular}{|c|c|c|c|c|}
\hline & IV-cb $(n=142)$ & $\mathbf{I V}+\mathbf{c b}(\mathbf{n}=\mathbf{1 2 3})$ & $\mathbf{B V}(\mathbf{n}=\mathbf{7 0})$ & $P$ value \\
\hline Operative time (mean $\pm \mathrm{SD}$ ) (min) & $58.4 \pm 10.2$ & $58.1 \pm 12.3$ & $118.4 \pm 17.6$ & $<.001^{*}$ \\
\hline Blood loss $($ mean $\pm \mathrm{SD})(\mathrm{mL})$ & $23.0 \pm 70.3$ & $15.0 \pm 21.9$ & $27.7 \pm 18.0$ & .179 \\
\hline Postoperative pain score, median (IQR) & $5(4-5)$ & $5(4-5)$ & $6(5-6)$ & $<.001^{*}$ \\
\hline Postoperative length of stay (mean $\pm \mathrm{SD}$ ) (d) & $5.6 \pm 2.4$ & $5.5 \pm 2.4$ & $7.1 \pm 3.1$ & $<.001^{*}$ \\
\hline Complication, $\%(n)$ & $11(15)$ & $14(17)$ & $16(11)$ & .527 \\
\hline Contralateral recurrence, $\%(n)$ & $0.7(1)$ & $14.6(18)$ & $2.9(2)$ & .002 \\
\hline Ipsilateral recurrence, $\%(n)$ & $8.5(12)$ & $8.1(10)$ & $7.1(5)$ & \\
\hline Follow-up, median (IQR) (mo) & $71(52-96)$ & 77 (40-97) & $74(57-104)$ & .189 \\
\hline
\end{tabular}

$I V-c b$, Ipsilateral video-assisted thoracic surgery without contralateral blebs/bullae; $I V+c b$, ipsilateral video-assisted thoracic surgery with contralateral blebs/bullae; $B V$, bilateral video-assisted thoracic surgery with contralateral blebs/bullae; $S D$, standard deviation; $I Q R$, interquartile range. *Significance after posterior comparison with Bonferroni correction $(\mathrm{BV}>\mathrm{IV}-\mathrm{cb}, \mathrm{BV}>\mathrm{IV}+\mathrm{cb})$.

recommended operation on PSP regardless of whether blebs/bullae are discovered by HRCT, as long as surgical indications hold. ${ }^{17}$

Early surgery for PSP to prevent ipsilateral recurrence has been advocated in certain high-risk patients exhibiting blebs/bullae on HRCT. ${ }^{4,16-18}$ However, there is still a lack of consensus of optimal treatment for the patients with radiographically demonstrated contralateral blebs/bullae. In our study, we compared the pro and cons between patients receiving ipsilateral VATS and bilateral VATS in terms of the presence and absence of contralateral blebs/ bullae. The patient characteristics were similar and comparable. The perioperative data disclosed significant increases in operative time, postoperative pain score, and length of hospital stay in group BV, which were due to the sequential bilateral similar procedures performed under the same single anesthesia. Despite these statistical differences, the postoperative complications among groups were revealed to be statistically insignificant. Therefore, we considered it safe for inherently young and physiologically fit patients to undergo 1-stage operation. The patients and their families were satisfied with the outcome with regard to both cosmetic aspect and recurrence prevention.

Another controversial issue is the correlation between risk factors and pneumothorax recurrence. Cardillo and col-

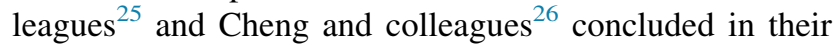
studies that smoking was associated with a significant effect on the recurrence rate of PSP. On the other hand, factors such as younger age, gender, prolonged air leakage, low BMI, and presence of blebs/bullae on HRCT also play an important role in the development of recurrence. ${ }^{27-30}$ Moreover, Huang and colleagues ${ }^{8}$ reported that the presence of contralateral blebs/bullae and being underweight $\left(\mathrm{BMI}<18.5 \mathrm{~kg} / \mathrm{m}^{2}\right)$ are independent risk factors for contralateral recurrence. ${ }^{10}$ On the contrary, we did not find any statistical differences regarding these factors, including female gender, lower BMI, and smoking in our study. It is noteworthy that the percentage of smokers in our series is relatively low (72/335 patients, $21.5 \%$ ) compared with

TABLE 3. Univariate analysis of risk factors for recurrence

\begin{tabular}{|c|c|c|c|c|}
\hline & $\begin{array}{l}\text { Contralateral recurrence } \\
\qquad(\mathbf{n}=\mathbf{2 1})\end{array}$ & $\begin{array}{l}\text { Ipsilateral recurrence } \\
\qquad(\mathbf{n}=\mathbf{2 7})\end{array}$ & No recurrence $(n=287)$ & $P$ value \\
\hline Age (y) & $20.6 \pm 6.2$ & $18.0 \pm 3.4$ & $21.7 \pm 5.6$ & .003 \\
\hline BMI $\left(\mathrm{kg} / \mathrm{m}^{2}\right)$ & $19.6 \pm 1.7$ & $18.7 \pm 2.1$ & $19.5 \pm 2.2$ & .169 \\
\hline \multicolumn{5}{|l|}{ Gender } \\
\hline Female & $3(14)$ & $1(4)$ & $37(13)$ & \\
\hline Male & $18(86)$ & $26(96)$ & $250(87)$ & .363 \\
\hline \multicolumn{5}{|l|}{ Smoking } \\
\hline No & $17(81)$ & $24(89)$ & $222(77)$ & \\
\hline Yes & $4(19)$ & $3(11)$ & $65(23)$ & .056 \\
\hline \multicolumn{5}{|l|}{ Treatment } \\
\hline IV-cb & $1(5)$ & $12(44)$ & $129(45)$ & \\
\hline $\mathrm{IV}+\mathrm{cb}$ & $18(86)$ & $10(37)$ & $95(33)$ & \\
\hline $\mathrm{BV}$ & $2(9)$ & $5(19)$ & $63(22)$ & $<.001$ \\
\hline
\end{tabular}

Data are presented as No. $(\%) . B M I$, Body mass index; $I V$ - $c b$, ipsilateral video-assisted thoracic surgery without contralateral blebs/bullae; $I V+c b$, ipsilateral video-assisted thoracic surgery with contralateral blebs/bullae; $B V$, bilateral video-assisted thoracic surgery with contralateral blebs/bullae. 


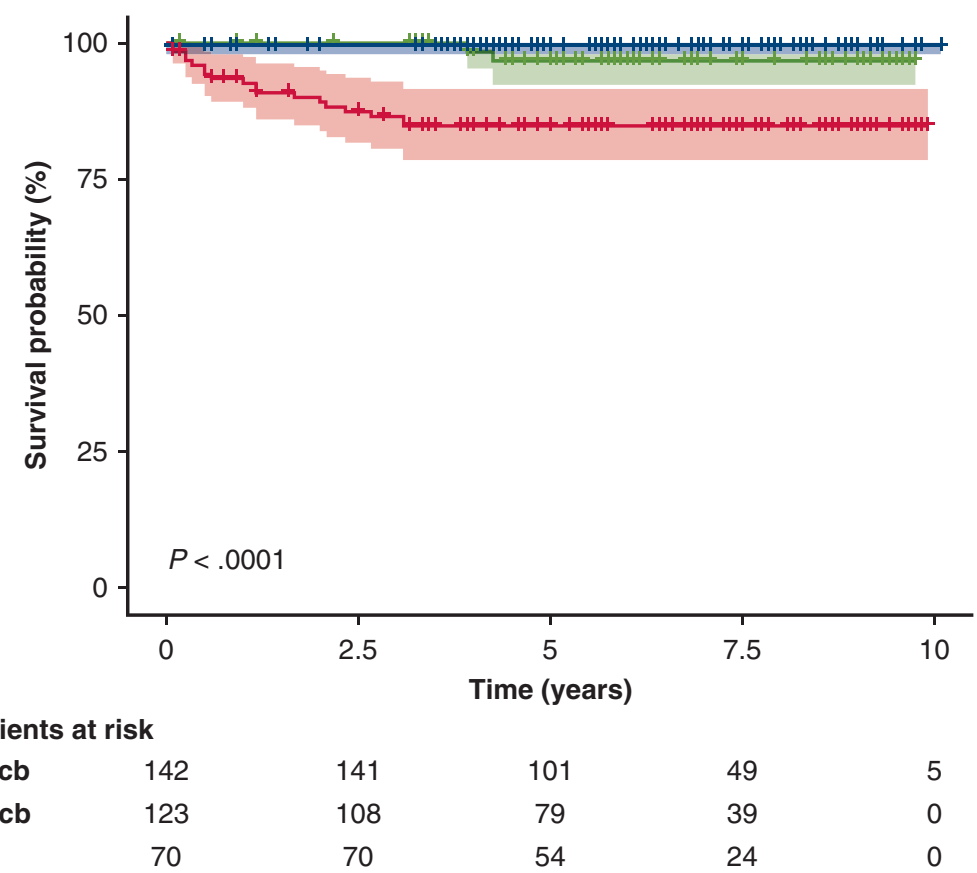

FIGURE 4. Kaplan-Meier curves demonstrating contralateral recurrence-free survival of patients with PSP who were treated in group IV-cb, IV+cb, or BV. IV-c $b$, Ipsilateral video-assisted thoracic surgery without contralateral blebs/bullae; $I V+c b$, ipsilateral video-assisted thoracic surgery with contralateral blebs/bullae; $B V$, bilateral video-assisted thoracic surgery with contralateral blebs/bullae.

that reported in the literature, which may be due to the unique culture of Taiwan. Nevertheless, our univariate and multivariate analyses showed that young age ( $<18$ years) and group IV $+\mathrm{cb}$ bear significant difference for contralateral recurrence $(P=.024, P=.003$, respectively). The result is comparable to the finding of a high incidence of recurrence in adolescents based on the nationwide population-based study ${ }^{1}$ and our previous research, emphasizing contralateral blebs intervention. ${ }^{9}$

\section{Study Strengths and Limitations}

In addition to the large collected databases and uniform treatment decisions based on our protocol, established since 2006 concerning patients with PSP with contralateral blebs/ bullae, ${ }^{9}$ a key strength in our study is that there were comparably long-term follow-up periods among groups (median, 75; IQR, 50-99 months). Through the Kaplan-Meier curve, the majority of contralateral recurrence $(14 / 21 ; 66.7 \%)$ and ipsilateral recurrence $(20 / 27 ; 74 \%)$ were observed to occur in the first 2 years after surgery. Further, all the overall recurrences (contralateral and ipsilateral) occurred within 5 years, except for 1 patient in group BV who experienced ipsilateral recurrence at 68 months (Figure E5). Although the current study presented a large number of cases with long-standing follow-ups, the main limitation was still its retrospective study design without randomization. Some selection bias does inevitably exist, including HRCT interpretation for blebs/bullae, patient's or family's attitude toward bilateral surgery, and operative technique between different surgeons.

With continued advances in minimally invasive thoracic surgery and the introduction of enhanced recovery protocols, ${ }^{31}$ single-stage bilateral VATS appears to be feasible

\section{CT scans of group IV-cb}

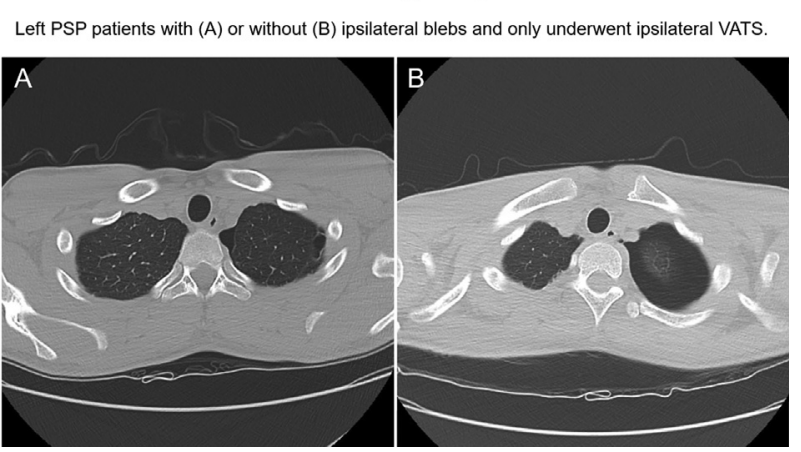

VIDEO 1. Simultaneous bilateral video-assisted thoracic surgery blebectomy reduces contralateral recurrence in patients operated for ipsilateral primary spontaneous pneumothorax. Video available at: https://www. jtcvs.org/article/S0022-5223(19)31723-4/fulltext. 
and beneficial for select patients with PSP involving contralateral pulmonary blebs/bullae. It circumvents the second hospitalization and reduces medical expenditures. ${ }^{9,22,24}$ We believe the number of institutions performing simultaneous bilateral VATS is growing without compromising patients' safety.

\section{CONCLUSIONS}

One-stage bilateral VATS for ipsilateral PSP with contralateral blebs/bullae not only exempts the future contralateral recurrence but also lessens potential psychologic and socioeconomic burden (Video 1).

\section{Conflict of Interest Statement}

Authors have nothing to disclose with regard to commercial support.

The authors thank Dr Chieh-Ni Kao for data collection and Jadzia Chou for language revision.

\section{References}

1. Huang YH, Chang PY, Wong KS, Chang CJ, Lai JY, Chen JC. An age-stratified longitudinal study of primary spontaneous pneumothorax. J Adolesc Health. 2017;61:527-32.

2. MacDuff A, Arnold A, Harvey J, BTS Pleural Disease Guideline Group. Management of spontaneous pneumothorax: British Thoracic Society Pleural Disease Guideline 2010. Thorax. 2010;65(Suppl 2):ii18-31.

3. Baumann MH, Strange C. Treatment of spontaneous pneumothorax: a more aggressive approach? Chest. 1997;112:789-804.

4. Casali C, Stefani A, Ligabue G, Natali P, Aramini B, Torricelli P, et al. Role of blebs and bullae detected by high-resolution computed tomography and recurrent spontaneous pneumothorax. Ann Thorac Surg. 2013;95:249-55.

5. Martínez-Ramos D, Angel-Yepes V, Escrig-Sos J, Miralles-Tena JM, SalvadorSanchís JL. Usefulness of computed tomography in determining risk of recurrence after a first episode of primary spontaneous pneumothorax: therapeutic implications. Arch Bronconeumol. 2007;43:304-8.

6. Ouanes-Besbes L, Golli M, Knani J, Dachraovi F, Nciri N, EI Atrous S, et al. Prediction of recurrent spontaneous pneumothorax: CT scan findings versus management features. Respir Med. 2007;101:230-6.

7. Sihoe AD, Yim AP, Lee TW, Wan S, Yuen EH, Wan IY, et al. Can CT scanning be used to select patients with unilateral primary spontaneous pneumothorax for bilateral surgery? Chest. 2000;118:380-3.

8. Huang TW, Lee SC, Cheng YL, Tzao C, Hsu HH, Chang H, et al. Contralateral recurrence of primary spontaneous pneumothorax. Chest. 2007;132:1146-50.

9. Chou SH, Li HP, Lee JY, Chang SJ, Lee YL, Chang YT, et al. Is prophylactic treatment of contralateral blebs in patients with primary spontaneous pneumothorax indicated? J Thorac Cardiovasc Surg. 2010;139:1241-5.

10. Chen YY, Huang HK, Chang H, Lee SC, Huang TW. Postoperative predictors of ipsilateral and contralateral recurrence in patients with primary spontaneous pneumothorax. J Thorac Dis. 2016;8:3217-24.

11. Lang-Lazdunski L, de Kerangal X, Pons F, Jancovici R. Primary spontaneous pneumothorax: one-stage treatment by bilateral videothoracoscopy. Ann Thorac Surg. 2000;70:412-7.

12. Schramel FM, Postmus PE, Vanderschueren RG. Current aspects of spontaneous pneumothorax. Eur Respir J. 1997;10:1372-9.
13. Sadikot RT, Greene T, Meadows K, Arnold AG. Recurrence of primary spontaneous pneumothorax. Thorax. 1997;52:805-9.

14. Baumann MH, Strange C, Heffner JE, Light R, Kirby TJ, Klein J, et al. Management of spontaneous pneumothorax: an American College of Chest Physicians Delphi consensus statement. Chest. 2001;119:590-602.

15. Tschopp JM, Bintcliffe O, Astoul P, Canalis E, Driesen P, Janssen J, et al. ERS task force statement: diagnosis and treatment of primary spontaneous pneumothorax. Eur Respir J. 2015;46:321-35.

16. Chou SH, Cheng YJ, Kao EL. Is video-assisted thoracic surgery indicated in the first episode primary spontaneous pneumothorax. Interact Cardiovasc Thorac Surg. 2003;2:552-4.

17. Kim JT, Oh TY, Chang WH, Kong JH, Baek KS, Lee WJ, et al. Natural course of spontaneous pneumothorax without bullae or blebs under high-resolution computed tomography. Thorac Cardiovasc Surg. 2014;62:505-8.

18. Primavesi F, Jäger T, Meissnitzer T, Buchner S, Reich-Weinberger S, Öfner D et al. First episode of spontaneous pneumothorax: CT-based scoring to select patients for early surgery. World J Surg. 2016;40:1112-20.

19. Baronofsky ID, Warden HG, Kaufman JL, Whatley J, Hanner JM Bilateral therapy for unilateral spontaneous pneumothorax. J Thorac Surg. 1957;34:310-9.

20. Ayed AK. Bilateral video-assisted thoracoscopic surgery for bilateral spontaneous pneumothorax. Chest. 2002;122:2234-7.

21. Wu YC, Chu Y, Liu YH, Yeh CH, Chen TP, Liu HP. Thoracoscopic ipsilateral approach to contralateral bullous lesion in patients with bilateral spontaneous pneumothorax. Ann Thorac Surg. 2003;76:1665-7.

22. Wang X, Wang L, Wang H, Zhang H. Simultaneous bilateral video-assisted thoracoscopic surgery for the treatment of primary spontaneous pneumothorax. Cell Biochem Biophys. 2015;71:1703-8.

23. Li X, Wang X, Zhang H, Cheng H, Cao Q. Unilateral single-port thoracoscopic surgery for bilateral pneumothorax or pulmonary bullae. J Cardiothorac Surg. 2019;14:71.

24. Guo Z, Yin W, Zhang X, Xu X, Liu H, Shao W, et al. Primary spontaneous pneumothorax: simultaneous treatment by bilateral non-intubated videothoracoscopy. Interact Cardiovasc Thorac Surg. 2016;23:196-201.

25. Cardillo G, Bintcliffe OJ, Carleo F, Carbone L, Di Martino M, Kahan BC, et al. Primary spontaneous pneumothorax: a cohort study of VATS with talc poudrage. Thorax. 2016;71:847-53

26. Cheng YL, Huang TW, Lin CK, Lee SC, Tzao C, Chen JC, et al. The impact of smoking in primary spontaneous pneumothorax. J Thorac Cardiovasc Surg. 2009; 138:192-5.

27. Tan J, Yang Y, Zhong J, Zuo C, Tang H, Zhao H, et al. Association between BM and recurrence of primary spontaneous pneumothorax. World J Surg. 2017;41: 1274-80.

28. Imperatori A, Rotolo N, Spagnoletti M, Festi L, Berizzi F, Di Natale D, et al. Risk factors for postoperative recurrence of spontaneous pneumothorax treated by video-assisted thoracoscopic surgery. Interact Cardiovasc Thorac Surg. 2015 20:647-51.

29. Hofmann HS, Suttner T, Neu R, Potzger T, Szöke T, Grosser C, et al. Burden between undersupply and overtreatment in the care of primary spontaneous pneumothorax. Thorac Cardiovasc Surg. 2018;66:575-82.

30. Nakayama T, Takahashi Y, Uehara H, Matsutani N, Kawamura M Outcome and risk factors of recurrence after thoracoscopic bullectomy in young adults with primary spontaneous pneumothorax. Surg Today. 2017; 47:859-64.

31. Jones NL, Edmonds L, Ghosh S, Klein AA. A review of enhanced recovery for thoracic anaesthesia and surgery. Anaesthesia. 2013;68:179-89.

Key Words: primary spontaneous pneumothorax, contralateral blebs/bullae, recurrence, one-stage VATS, simultaneous contralateral blebectomy 

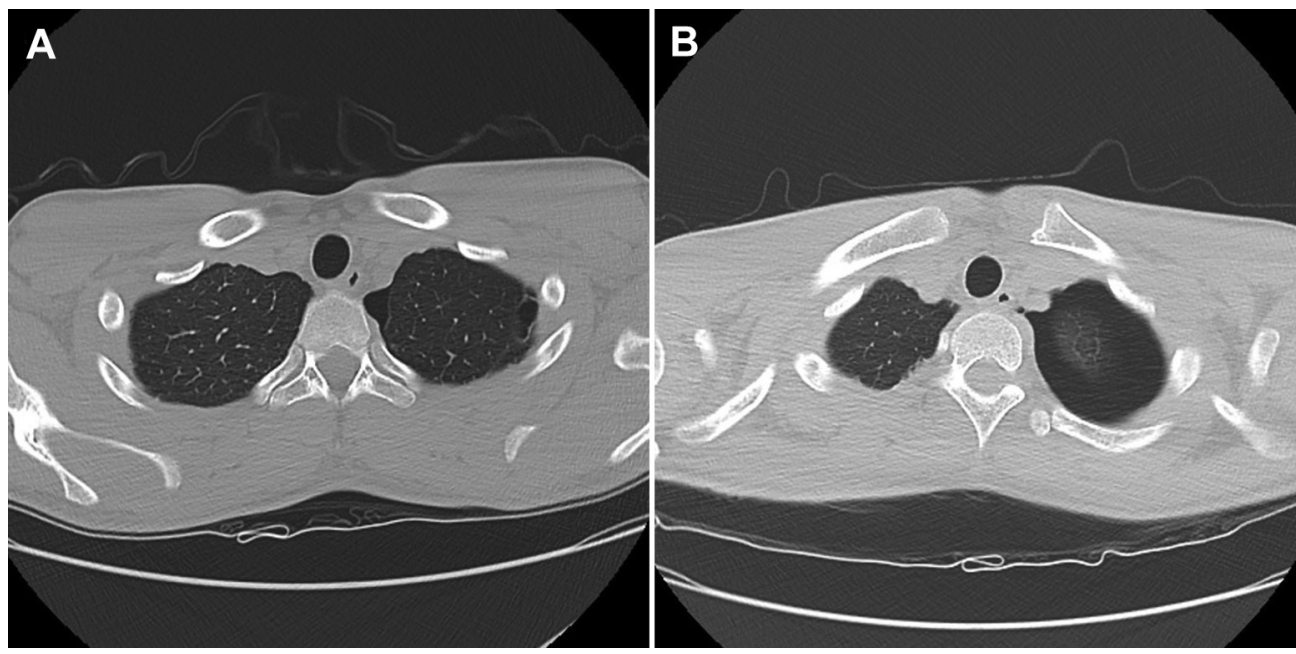

FIGURE E1. Computed tomography showing a patient with left spontaneous pneumothorax with (A) or without ipsilateral blebs (B) who underwent ipsilateral VATS.
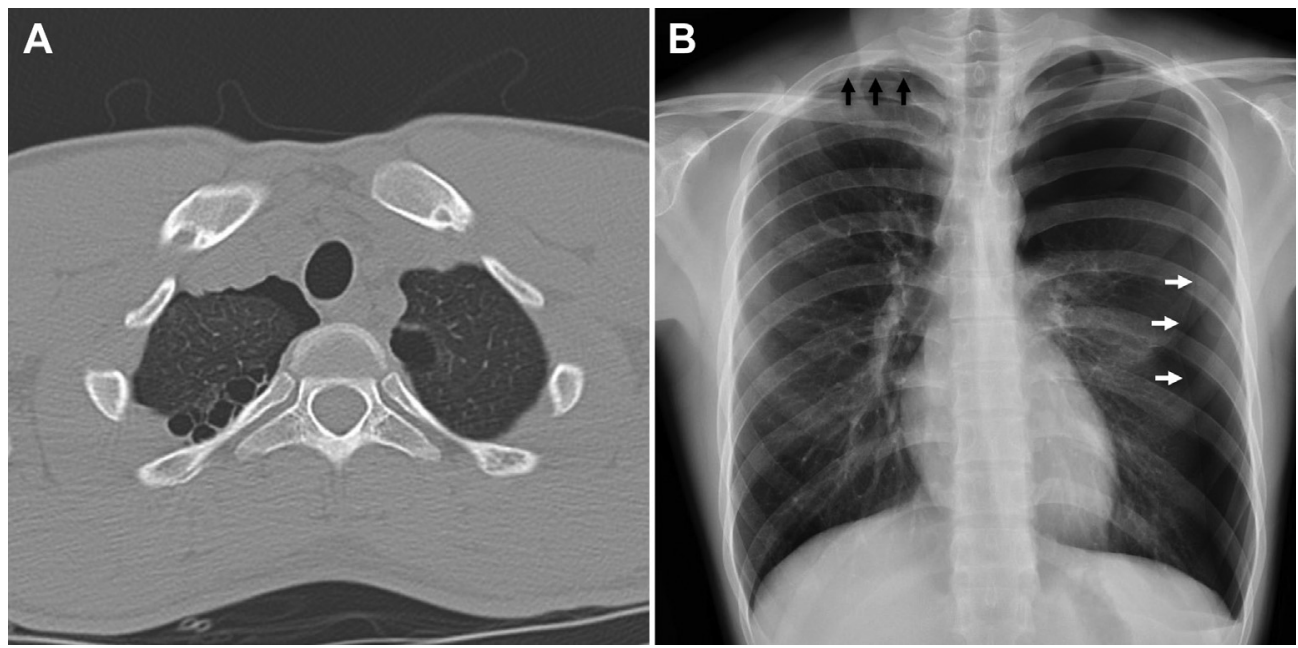

FIGURE E2. Computed tomography showing a patient with right spontaneous pneumothorax and bilateral blebs (A) who underwent ipsilateral VATS and developed a later contralateral pneumothorax (B) (black arrows: previous surgical staple lines and clips; white arrows: visceral pleural line). 

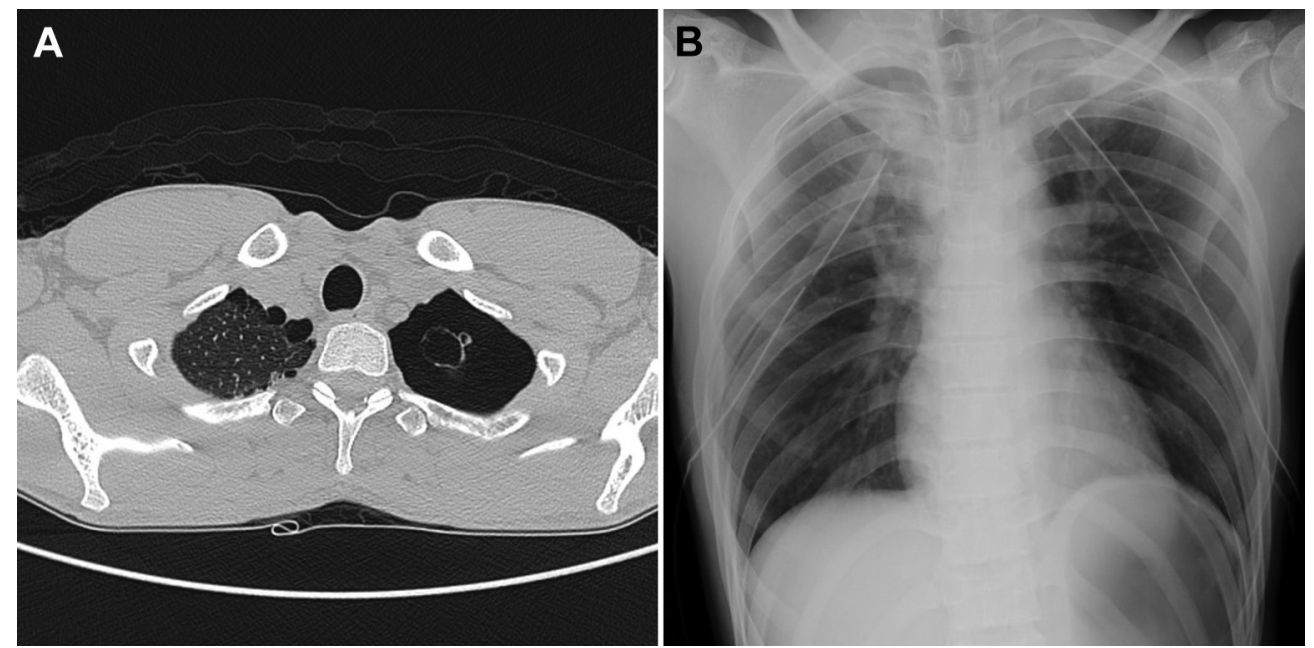

FIGURE E3. Computed tomography showing a patient with left spontaneous pneumothorax with bilateral blebs (A) who underwent 1-stage bilateral VATS (B).

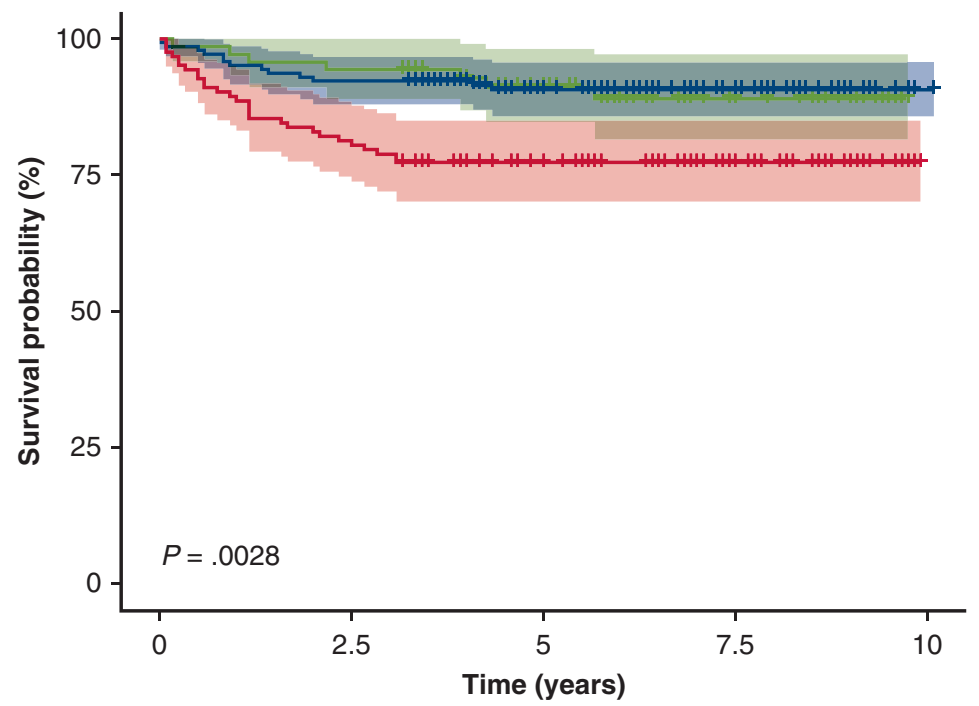

Patients at risk

$\begin{array}{lccccc}\text { IV-cb } & 142 & 131 & 90 & 46 & 3 \\ \text { IV+cb } & 123 & 99 & 74 & 38 & 0 \\ \text { BV } & 70 & 66 & 52 & 23 & 0\end{array}$

FIGURE E4. Kaplan-Meier curves showing overall recurrence-free survival of patients with PSP who were treated in group IV-cb, IV+cb, or BV. $I V$ - $c b$, Ipsilateral video-assisted thoracic surgery without contralateral blebs/bullae; $I V+c b$, ipsilateral video-assisted thoracic surgery with contralateral blebs/bullae; $B V$, bilateral video-assisted thoracic surgery with contralateral blebs/bullae. 


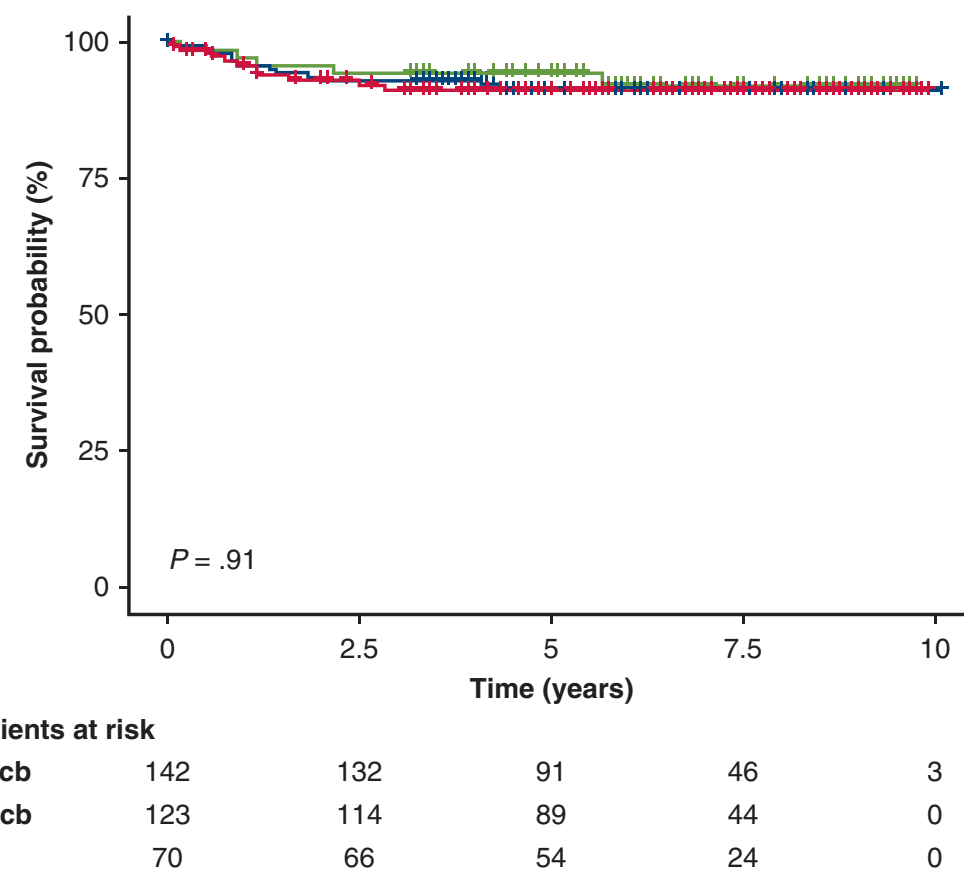

FIGURE E5. Kaplan-Meier curves showing ipsilateral recurrence-free survival of patients with PSP who were treated in group IV-cb, IV +cb, or BV. IV-cb, Ipsilateral video-assisted thoracic surgery without contralateral blebs/bullae; $I V+c b$, ipsilateral video-assisted thoracic surgery with contralateral blebs/ bullae; $B V$, bilateral video-assisted thoracic surgery with contralateral blebs/bullae.

TABLE E1. Risk of recurrence based on patient characteristics and treatment groups

\begin{tabular}{|c|c|c|c|c|c|c|}
\hline & Contralateral, \% (n) & HR $(95 \% \text { CI })^{*}$ & $P$ value* & Ipsilateral, \% (n) & HR $(95 \% \text { CI })^{*}$ & $P$ value ${ }^{*}$ \\
\hline \multicolumn{7}{|l|}{ Age (y) } \\
\hline$\geq 18$ & $4.8(12 / 251)$ & 1.0 & & $4.8(12 / 251)$ & 1.0 & \\
\hline$<18$ & $10.7(9 / 84)$ & $2.71(1.14-6.44)$ & .024 & $17.8(15 / 84)$ & $4.29(2.01-9.17)$ & .0002 \\
\hline \multicolumn{7}{|c|}{ BMI $\left(\mathrm{kg} / \mathrm{m}^{2}\right)$} \\
\hline$\geq 18.5$ & $6.8(15 / 221)$ & 1.0 & & $8.1(18 / 221)$ & 1.0 & \\
\hline$<18.5$ & $5.3(6 / 114)$ & $0.75(0.29-1.93)$ & .549 & $7.9(9 / 114)$ & $0.94(0.42-2.09)$ & .872 \\
\hline \multicolumn{7}{|l|}{ Gender } \\
\hline Female & $7.3(3 / 41)$ & 1.0 & & $2.4(1 / 41)$ & 1.0 & \\
\hline Male & $6.1(18 / 294)$ & $0.88(0.26-2.99)$ & .838 & $8.8(26 / 294)$ & $0.27(0.04-1.97)$ & .194 \\
\hline \multicolumn{7}{|l|}{ Smoking } \\
\hline No & $6.5(17 / 263)$ & 1.0 & & $9.1(24 / 263)$ & 1.0 & \\
\hline Yes & $5.5(4 / 72)$ & $0.79(0.27-2.36)$ & .679 & $4.2(3 / 72)$ & $0.43(0.13-1.45)$ & .176 \\
\hline \multicolumn{7}{|l|}{ Treatment } \\
\hline IV-cb & $0.7(1 / 142)$ & 1.0 & & $8.5(12 / 142)$ & 1.0 & \\
\hline $\mathrm{IV}+\mathrm{cb}$ & $14.6(18 / 123)$ & $22.13(2.96-165)$ & .003 & $8.1(10 / 123)$ & $1.13(0.49-2.61)$ & .777 \\
\hline $\mathrm{BV}$ & $2.9(2 / 70)$ & $3.81(0.35-42.00)$ & .275 & $7.1(5 / 70)$ & $0.84(0.30-2.39)$ & .748 \\
\hline
\end{tabular}

HR, Hazard ratio; $C I$, confidence interval; $B M I$, body mass index; $I V-c b$, ipsilateral video-assisted thoracic surgery without contralateral blebs/bullae; $I V+c b$, ipsilateral videoassisted thoracic surgery with contralateral blebs/bullae; $B V$, bilateral video-assisted thoracic surgery with contralateral blebs/bullae. $*$ The HRs and $P$ value were estimated by a multivariable Cox regression model. 